\title{
Measurement of lingual vibrotactile sensitivity using one-trial and three-trial threshold criteria*
}

\author{
KAL M. TELAGE \\ Department of Speech Pathology and Audiology, State University of New York at Geneseo, Geneseo, New York 14454 \\ and \\ DONALD J. FUCCI \\ School of Hearing and Speech Sciences, Ohio University, A thens, Ohio 45701
}

\begin{abstract}
Ascending lingual vibrotactile thresholds were obtained from the anterior midline of the tongue for 110 normal speaking adult Ss at 200 and $400 \mathrm{~Hz}$. Two threshold criteria, one test trial and the median of three successive trials, were used at each frequency. The t test comparisons between testing modes showed no significant differences. Correlations between testing modes were high and suggested direct rank orderings of threshold values. These findings were discussed in terms of possible advantages of using the simpler procedure as a more practical approach for measuring oral tactile sensation with children.
\end{abstract}

An increasing body of research evidence derived from neurophysiological research has promoted a closed-loop feedback theory for explaining the control of dynamic coarticulatory events (Silverman, 1961; Mountcastle \& Darian-Smith, 1968; Sussman, 1972). In such a scheme, the mediating role is performed by neural receptors in the tongue and other oral structures which are capable of transmitting complex spatial and temporal information. The tongue possesses two feedback systems which have this capacity; the muscle-spindle and end-organ transducers, and the intricate exteroceptive touch endings (Sussman, 1972). Recent investigations, which have successfully employed vibration as a means for assessing oral tactile sensation, have provided information concerning the response characteristics of the latter neural elements (Fucci, Hall, \& Weiner, 1971; Fucci, 1972; Telage, Fucci, \& Arnst, 1972).

Subsequent research using oral vibrotactile stimulation has been directed toward developing a methodology which might form the basis for further experimental and diagnostic evaluations of tactile feedback mechanisms subserving the speech function. The initial focus concerned investigating psychophysical parameters involved in presenting the vibratory signal to the midline of the anterior lingual surface. Hall, Fucci, and Arnst (1972) found no differences between data obtained with either the method of limits or the method of adjustment. Differences between ascending and descending thresholds obtained with pulsed stimulation were also found to be insignificant. However, an ascending procedure was recommended for use in threshold testing to avoid any possible adaptation effects.

Fucci, Arnst, and Telage (1972) furthered this earlier finding by demonstrating that a critical parameter affecting vibrotactile thresholds obtained from the

\footnotetext{
*Sponsored by James L. Bruning, who takes full editorial responsibility for its contents.
}

tongue is pulsed, versus continuous stimulation. Their comparative investigation showed significantly greater tactile sensitivity for Ss when thresholds were obtained using a pulsed vibratory signal. This result was similar to data reported by Hirsh (1952) for auditory thresholds.

In a follow-up study Fucci, Arnst, Telage, and McCaffrey (1973) evaluated oral tactile sensitivity for Ss using continuous vibratory stimuli in ascending and descending presentations. The significantly poorer descending threshold responses provided a preliminary basis for subsequent research into the effects of lingual tactile adaptation.

As a further attempt to define appropriate methods for measuring vibrotactile sensation, the present study was designed to compare threshold responses obtained using a one-trial procedure with those determined from the median of three separate trials. Although vibrotactile investigations have traditionally accepted the median of three test trials as the threshold criterion, this testing procedure is time consuming and generally increases the possibility that $\mathrm{S}$ fatigue may distort the results when working with the tongue.

\section{METHOD}

The present study included 110 Ss ranging in age from 18 to 36 years, none of whom reported a history of speech defects and sensory or motor impairments. Lingual vibrotactile thresholds were obtained for all Ss at 200 and $400 \mathrm{~Hz}$, using an ascending method of limits. Two separate thresholds were determined at each of the frequencies used. The criteria used to establish threshold were a single test trial and the median of three test trials.

The equipment used in this study has been described in a previous publication (Telage, Fucci, \& Arnst, 1972). The stimulus unit included a sine-wave generator, frequency counter, electronic switch (designed to maintain a sine-wave configuration), interval timer, amplifier, variable attenuator, and electromagnetic vibrator. The pulsed vibratory signal generated had a $50 \%$ duty cycle (on $1 \mathrm{sec}$ and off $1 \mathrm{sec}$ ) with a rise and decay time of $100 \mathrm{msec}$. The measurement unit consisted of an accelerometer, cathode follower, microphone amplifier, and 
Table 1

A Comparison of Mean Differences in Microns of Displacement for Vibrotactile Thresholds Obtained Using One-Trial and Three-Trial Procedures

\begin{tabular}{lccrrr}
\hline \multirow{2}{*}{$\begin{array}{c}\text { Threshold } \\
\text { Criteria }\end{array}$} & \multicolumn{2}{c}{$200 \mathrm{~Hz}$} & & \multicolumn{2}{c}{$400 \mathrm{~Hz}$} \\
\cline { 2 - 3 } \cline { 5 - 6 } & 1 & $\mathrm{M} / 3$ & & 1 & $\mathrm{M} / 3$ \\
\hline Mean & .301 & .305 & & .209 & .208 \\
SD & .155 & .168 & & .094 & .102 \\
$\mathrm{t}$ & \multicolumn{2}{c}{$-0.48^{*}$} & & \multicolumn{2}{c}{$0.06^{*}$} \\
\hline
\end{tabular}

Note $-N=110$

*Nonsignificant at the .01 level of confidence.

vacuum tube voltmeter. A white-noise generator was used to present auditory masking at $100-110 \mathrm{~dB}$ SPL to Ss through TDH-39 headphones.

Ss were seated in a dental chair and asked to extend the tongue between two sterilized plastic disks. The tongue was clamped in place tightly enough to hold it yet not enough to affect normal circulation or place undue pressure on the structure. A hole in the top disk provided the probe with access to the anterior midline surface of the tongue. The contactor area at the end of the probe was $.128 \mathrm{~cm}^{2}$ with a free surround area of $.310 \mathrm{~cm}^{2}$.

To maintain constant pressure of the vibrator on the tongue for all Ss, the probe was lowered until a voltmeter recorded contact. The negative wire from the voltmeter was connected to the base of the electromagnetic vibrator and the positive wire was held between S's thumb and forefinger. When contact was established with the tongue, the voltmeter registered a needle deflection. The probe was then lowered $1 \mathrm{~mm}$ further into the tongue.

Ss were required to depress an indicator switch as soon as they detected the vibratory stimulus. All thresholds were initially recorded in millivolts and later changed to displacement in microns using a standard $\mathrm{g}$ formula for acceleration conversion.

\section{RESULTS AND DISCUSSION}

Vibrotactile threshold means and standard deviations were determined for data obtained using both testing criteria at 200 and $400 \mathrm{~Hz}$, respectively. These results appear in Table 1. Both threshold methods resulted in very similar mean threshold values at each frequency. Standard deviations reflected little inter-S variability relative to the means.

Statistical analysis for significant differences between

Table 2

Reliability Coefficients for One-Trial and Three-Trial Threshold Procedures

\begin{tabular}{ccc}
\hline Frequency & $\mathrm{r}$ \\
\hline $200 \mathrm{~Hz}$ & $(\mathrm{~N}=110)$ & $.85^{*}$ \\
$400 \mathrm{~Hz}$ & $.82^{*}$ \\
\hline
\end{tabular}

*Significant at the .01 level of confidence. the threshold means for both testing procedures was performed using $t$ tests for related measures. It can be seen from the data in Table 1 , that very small $t$ ratios indicate nonsignificant differences.

Pearson product correlations were computed as direct measures of the covariance between the one trial and three trial threshold methods. Table 2 shows high positive correlation coefficients at each frequency. These coefficients indicate moderately strong relationships which suggest similar rank orderings of the threshold values for both techniques.

It appears from the data that a single threshold trial can yield results which are not significantly different from those obtained employing the median of three successive trials. Moreover, high intra-S reliability between procedures suggests that either method produces very similar threshold results for a given individual. These results provide some basis for further exploration of the one-trial testing method. Some advantages are that it could facilitate testing procedures in general and might provide a more feasible approach for measuring lingual vibrotactile sensation in children. A detailed discussion of clinical implications of the vibrotactile methodology is presented by Telage and Fucci (1973).

\section{REFERENCES}

Fucci, D. Oral vibrotactile sensation: An evaluation of nornial and defective speakers. Journal of Speech \& Hearing Resear $h$ 1972, 15, 179-184.

Fucci, D., Arnst, D., \& Telage, K. M. The effects of pulsed and continuous stimulation on vibrotactile thresholds obtained from the tongue. Psy chonomic Science, 1972, 29, 83-84.

Fucci, D., Arnst, D., Telage, K. M., \& McCaffrey, P. Measurement of lingual vibrotactile sensitivity using pulsed and continuous stimulation. Bulletin of the Psychonomic Society, 1973, 2, 85-86.

Fucci, D., Hall, D., \& Weiner, F. Normative study of oral and non-oral structures using vibrotactile stimuli. Perceptual \& Motor Skills, 1971, 33, 1099-1105.

Hall, D. Fucci, D., \& Arnst, D. Vibrotactile stimulation: An investigation of psychophysical methods for establishing thresholds. Perceptual \& Motor Skills, 1972, 34, 891-898.

Hirsh, I. J. The measurement of hearing. New York: McGraw-Hill, 1952.

Mountcastle, V. B., \& Darian-Smith, I. Neural mechanisms in somethesis. In V. B. Mountcastle (Ed.), Medical physiology. (12th ed.) St. Louis: Mosley, 1968.

Silverman, S. I. Oral physiology. St. Louis: Mosley, 1961.

Sussman, H. M. What the tongue tells the brain. Psychological Bulletin, 1972, 77, 262-272.

Telage, K. M., \& Fucci, D. Vibrotactile stimulation: A future clinical tool for speech pathologists. Journal of Speech \& Hearing Disorders, 1973, 38, 442-447.

Telage, K. M., Fucci, D., \& Arnst, D. Normative study of oral vibrotactile sensitivity. Perceptual \& Motor Skills, 1972, 35, 671-676.

(Received for publication March 4, 1974.) 